\title{
Development and disease of the photoreceptor cilium
}

\author{
Vasanth Ramamurthy ${ }^{1,3}$ and Michel Cayouette ${ }^{1,2,3,4 *}$ \\ ${ }^{1}$ Institut de recherches cliniques de Montréal (IRCM), Montréal, QC, Canada \\ ${ }^{2}$ Département de Médecine, Université de Montréal, Montréal, QC, Canada \\ ${ }^{3}$ Division of experimental Medicine, McGill University, Montreal, QC, Canada \\ ${ }^{4}$ Department of Anatomy and Cell Biology, McGill University, Montreal, QC, Canada \\ * Corresponding author (michel.cayouette@ircm.qc.ca)
}

\section{ACKNOWLEDGMENTS}

We would like to thank Pierre Fabre and Jean-Francois Ouimette for comments on this manuscript. V. R. is supported by a studentship from the Foundation Fighting Blindness Canada (FFB-C). Work in M.C.'s lab is funded by the Canadian Institutes of Health Research (CIHR) and the FFB-C. M.C. is a CIHR New Investigator and a W.K. Stell Scholar of the FFBC. 


\begin{abstract}
Primary cilia are microtubule-rich hair-like extensions protruding from the surface of most post mitotic cells. They act as sensory organelles that help interpret various environmental cues. Mutations in genes encoding proteins involved in ciliogenesis or protein transport to the primary cilia lead to a wide variety of diseases commonly referred to as ciliopathies, which include primary ciliary dyskinesia, situs invertus, hydrocephalus, kidney diseases, respiratory diseases, and retinal degenerations. In the retina, the photoreceptor cells have a highly specialized primary cilium called the outer segment (OS), which is essential for photosensation. Development of the photoreceptor OS shares key regulatory mechanisms with ciliogenesis in other cell types. Accumulating evidence indicate that mutations that affect OS development and/or protein transport to the OS generally lead to photoreceptor degeneration, which can be accompanied by a range of other clinical manifestations due to the dysfunction of primary cilia in different cell types. Here, we review the general mechanisms regulating ciliogenesis, and present different examples of mutations affecting OS ciliogenesis and protein transport that lead to photoreceptor degeneration. Overall, we conclude that the genetic and molecular evidence accumulated in recent years suggest a clear link between the development and function of the primary cilium and various clinical conditions. Future studies aimed at uncovering the cellular and molecular mechanisms implicated in ciliogenesis in a wide variety of animal models should greatly increase our understanding of the pathophysiology of many human diseases, including retinal degenerations.
\end{abstract}




\section{INTRODUCTION}

In multicellular organisms, different cell types display a characteristic morphology perfectly adapted to perform a specific function. In the nervous system, for example, neurons have distinct cellular compartments called axons and dendrites that are essential to send and receive signals respectively. This characteristic morphology is acquired during development by the establishment of an axis of polarity in the developing cell that provides spatial cues for the formation of subcellular compartments (1).

One such compartment that has often been overlooked is the primary cilium. The primary cilium is a found on most postmitotic vertebrate cell types as hair-like protrusion that extends from the cell into the extracellular space. They are commonly referred to as the cellular antennae, enabling the cell to transmit and receive information with its environment. Primary cilia are believed to have evolved from cilia and flagella. Cilia, along with its ancestral cousin flagella, are ancient organelles that arose early on in evolution to provide motility to unicellular organisms. The motility function of cilia and flagella has been retained in some mammalian primary cilia. For example, motile cilia in the inner lining of the trachea participate in generating an extracellular fluid flow, and flagella in spermatozoa are essential for motility. With increasing complexity in higher vertebrates, however, some cilia became immotile to perform specialized sensory functions such as photo-, mechano-, and chemo-sensations $(2,3)$.

The photoreceptor cells of the vertebrate retina have a specialized function for vision: convert light signals into an electrical output, a process referred to as phototransduction. This electrical output is then processed by other retinal neurons and sent to the brain, where higher order cortical neurons interpret the visual cues. The molecular machinery necessary for phototransduction is housed in a highly specialized primary cilium dedicated to this process - the outer segment (OS). The OS is an extraordinary example of compartmentalization of specific machinery in order to optimize function. Not surprisingly, mutations that affect OS development and/or function can lead to severe photoreceptor degeneration that ultimately result in vision loss. 
In recent years, the primary cilium in various cell types has received increasing attention due to its dysfunction in a wide variety of human diseases ranging from situs invertus and renal cysts, to sensorineural defects (4). Here, we will review the current knowledge on the molecular mechanisms that regulate cilium development, or ciliogenesis, with a particular emphasis on the photoreceptor OS and how defects in the development and function of this specialized cilium can lead to retinal degeneration.

\section{STRUCTURE AND DEVELOPMENT OF THE PHOTORECEPTOR CILIUM}

In the mammalian retina, photoreceptor cells develop from a pool of dividing multipotent retinal progenitor cells. Upon exit form the cell cycle, the postmitotic photoreceptor cell differentiate and develop a highly polarized morphology. The synaptic terminal is found on the basal side, whereas the OS, which houses the phototransduction machinery, is found on the apical side (Fig. 1A). The OS is composed of disk membranes stacked on top of each other, which optimizes the total surface available to accommodate a maximum amount of the photopigment protein opsin, thereby providing extreme sensitivity to light (Fig. 1B). All the

proteins required for phototransduction are translated in the inner segment (IS), a different compartment of the photoreceptor cell that houses the translation machinery and other organelles (Fig. 1C). To reach the OS, proteins translated in the IS must be trafficked through the connecting cilium, which links the IS to the OS and constitutes a sort of highway for proteins travelling to and from the $\mathrm{OS}$. The $\mathrm{CC}$ of the photoreceptors has a stereotypical non-motile cilium structure. Like all other cilia it has a microtubule backbone, the axoneme, which forms the cytoskeleton core. It is anchored in the IS by the basal body (a modified centriole). The photoreceptor axoneme extends almost the entire length of the OS. It has a microtubule arrangement of $9+0$, common to non-motile cilia, with 9 pairs of doublet microtubules arranged in a circular fashion. More distally from the basal bodies and into the OS, the axoneme takes the conformation of a $9+0$ singlet microtubule arrangement. This singlet conformation extends through much of the OS (Fig. 1B). The distal singlet microtubule conformation resembles primary cilium in the mammalian kidney and the C.elegans sensory cilia $(5,6)$. 
The photoreceptor OS develops initially by growing the $\mathrm{CC}$ at the apical end of the cell. This is followed by addition of disk membranes to the distal end of the $\mathrm{CC}$, forming the base of the OS. Disk membranes form by evagination of the plasma membrane at the apical end of the $\mathrm{CC}$ and pinch off to form the OS (Fig. 1C). In vertebrate cone photoreceptors the disk membranes are continuous with the plasma membrane, whereas in rod photoreceptors, the disks are separate from the plasma membrane. The disk membranes themselves contain different domains with a flat lamellar region in the middle, which houses the visual pigment opsin, and spherical disk region at the edge of the disks that contains other phototransduction proteins and structural proteins (Fig. 1B).

The process of disk membrane formation is maintained in the fully developed adult OS, which undergoes a continuous process of renewal. The disks at the most apical end of the OS are shed and engulfed by the retinal pigment epithelial cells bordering the OS, whereas new disks are added at the base of the OS. This process depends on the diurnal light cycle and has developed to clear out toxic byproducts of the phototransduction mechanism from the cell (7). This process of renewal necessitates a higher rate of delivery of newly synthesized proteins to the newly forming disks than when compared to simple protein turnover in other cell types. For example, 2000 opsin molecules are transported every minute from the IS to the OS (5). Hence, protein trafficking mechanisms are not only relevant during development of the OS, but also in mature photoreceptors.

\section{PROTEIN TRANSPORT IN CILIOGENESIS}

In all ciliated cells, primary cilia lack biosynthesis machinery. This means that the molecular components of the cilium are synthesized entirely in the cell and then transported into the cilium. This process, called intraflagellar transport (IFT), was first discovered in the unicellular organism Chlamydomonas $(8,9)$. IFT involves the transport of cargo associated with large protein complexes (IFT particles) along the axoneme by motor proteins. IFT particles act as "delivery trucks", picking up the cargo (axonemal subunits and structural proteins) at the base of the cilium and transporting them to the site of cilium synthesis, as it grows. To date, 17 IFT particles have been identified (10). They associate to form two multiprotein subcomplexes, IFT 
complex A and IFT complex B. The IFT particles also interact with motor proteins such as heterotrimeric kinesin 2 for anterograde transport towards the cilium, and dynein for retrograde transport towards the cell body. The IFT network is highly conserved among most species as well as within different cell types. In photoreceptors, cilium transport is complicated by the requirement of distinct trafficking machinery to target specific proteins to different domains of the OS. Once the CC is synthesized, OS proteins need to be sorted between the disk lamellar region, the disk rim region and the plasma membrane in the developing OS (Fig. 1B).

Many IFT particles have been reported to localize at the $\mathrm{CC}$ in vertebrate photoreceptors. Functional analysis of the IFT machinery comes from numerous knockout studies in mouse and zebrafish photoreceptors (5). In mouse mutants of the IFT particle, IFT88, OS show aberrant stacking of disk membranes and accumulation of opsin in the inner segment, which eventually becomes toxic to the cell and leads to photoreceptor cell death (11). In zebrafish mutants for IFT57, IFT88, and IFT172, photoreceptors are completely devoid of OS. Detailed analysis of OS development in these mutants revealed differences in the function of the three IFT particles. In the IFT57 mutant, for example, the OS develop normally, albeit shorter than in the wildtype, but eventually the photoreceptor starts accumulating opsin in the inner segment, leading to photoreceptor cell death (12). These results suggest that although IFT57 is not required for initial ciliogenesis, it is necessary for OS growth and maintenance. In contrast, no CC forms in the IFT88 and IFT172 mutants at the early stages of photoreceptor development, but disorganized disk membranes accumulate at the apical side of the IS, suggesting that IFT88 and IFT172 are required for the initial growth of the $\mathrm{CC}$, but are dispensable for OS disk membrane formation (10).

C.elegans studies have greatly helped our understanding of the mechanism of IFT motors. In C.elegans, the IFT machinery has two motors for anterograde transport: kinesin-2 (as in Chlamydomonas) and OSM-3. Kinesin-2 and OSM-3 work in tandem to transport IFT particles and their cargo in the middle section of doublet microtubules, whereas OSM-3 transports IFT particles in the more distal segment, where the axoneme layout is a $9+0$ circular arrangement of singlet microtubules $(13,14)$. The vertebrate ortholog of the anterograde motor protein kinesin-2 has been detected in the $\mathrm{CC}$ of mouse photoreceptors by electron microscopy. 
A photoreceptor-specific mouse mutant of a subunit of kinesin-2 (Kif3a) leads to accumulation of the phototransduction proteins opsin and arrestin in the cell body and IS of photoreceptors, which ultimately result in photoreceptor cell death $(15,16)$. These results suggest an important role for Kif3a in protein transport to the OS. Importantly, biochemical analyses have shown association of IFT particles with all three subunits of heterotrimeric kinesin-2 motor protein in retinal extracts, suggesting a conserved mechanisms for protein transport in the cilium form C. elegans to mammals $(17,18)$ (Fig. 2). The orthologue of OSM-3 in vertebrates, Kif17, has also been reported to localize at the $\mathrm{CC}$ in mouse and zebrafish photoreceptors (19), and Kif17 knockdown in zebrafish disrupts OS formation (20). These results suggest a role for this motor protein in the development of the photoreceptor OS, but it remains unclear whether Kif17 functions only in the distal segment of the cilium, as it does in C. elegans. Interestingly, primary cilium formation in kidney epithelial cells was not affected by Kif17 knockdown in zebrafish, suggesting a specific role in photoreceptor cilia. Together, these results provide an important conceptual advance suggesting that disrupted polarized trafficking of OS proteins to their specific cellular domain could generally lead to photoreceptor cell death, the common feature of retinal degenerative diseases. One potential mechanism for photoreceptor degeneration in such conditions is that the toxic byproducts of opsin cannot be cleared out easily from the cell body and IS trough the normal mechanism of phagocytosis by the pigmented epithelium (21) (Fig. 3).

\section{PHOTORECEPTOR CILIOPATHIES}

The fundamental studies described above suggest that, in humans, mutations in genes encoding proteins involved in ciliogenesis or protein transport to the OS would lead to photoreceptor degeneration. Since primary cilia have a critical role in a wide variety of cell types, it is also expected that photoreceptor degeneration would sometimes be accompanied by other clinical manifestations. Here, we will discuss different photoreceptor degenerative diseases in which mutations have been found in genes that are implicated in ciliogenesis and/or protein transport to the cilium (Table 1).

Retinitis Pigmentosa (RP) is a genetically heterogeneous group of disorders characterized by night blindness and progressive loss of peripheral vision due to rod photoreceptors 
undergoing cell death. Degeneration of rod photoreceptors is often followed by cone photoreceptor cell death, which eventually leads to loss of central vision. Although the majority of RP-causing mutations are found in genes that encode proteins of the phototransduction pathway (rhodopsin mutations, for example, account for up to $25 \%$ of autosomal-dominant RP cases $[22,23])$, many are found in genes that encode proteins involved in OS morphogenesis, ciliogenesis, and polarized protein transport to the cilium.

Mutations in the gene encoding the photoreceptor specific protein Retinitis Pigmentosa 1 (RP1), for example, have been associated with RP cases. In photoreceptor cells, the RP1 protein is localized along the axoneme and is especially concentrated at the site of OS disk membrane formation. Studies of RP1 mouse mutants revealed abnormal stacking and orientation of disk membranes, suggesting a role for RP1 in OS organization $(22,24)$. Interestingly, RP1 mouse mutants also show mislocalization of rhodopsin in the IS and cell body which, as we discussed above, is thought to result in photoreceptor degeneration. Although the precise role of RP1 in photoreceptors remain poorly understood, a recent study reported that RP1 binds to microtubules, suggesting that it might function by stabilizing the microtubules in the axoneme to regulate axonemal length and stability (25). Mutations in the $R D S$ gene, which encodes the glycoprotein rds/peripherin $(26,27)$, have also been associated to RP (reviewed in $(28,29)$ ). $\mathrm{Rds}$ /peripherin is localized to the rim of photoreceptor OS disk membranes, where it is thought to promote the normal flattening of the disk membranes (30). In rds/peripherin mutants, OS disk morphogenesis is disrupted, which lead to accumulation of rhodopsin in the IS and cell body and progressive photoreceptor cell death (31-33). The studies of both RP-1 and rds/peripherin demonstrate the importance of normal OS morphogenesis in photoreceptor cell survival, and raise the possibility that many unknown forms of RP might be associated to mutations in genes encoding proteins involved in OS morphogenesis.

Although the proteins discussed above appear to have only photoreceptor-specific roles, mutations in genes that encode proteins globally involved in ciliogenesis in various cell types can also lead to photoreceptor degeneration. In fact, retinal degeneration in the form of RP is a clinical phenotype of a number of human ciliopathies (Table 1). Ciliopathies is an emerging class of human disorders that are caused by dysfunction of the primary cilium. They can either involve 
single organ disorders or occur as multisystemic disorders with phenotypically variable and overlapping disease manifestations. The pleiotrophic nature of ciliopathies suggests that they are caused by disruption of global ciliogenesis and/or transport mechanisms. For example, Retinitis Pigmentosa GTPase Regulator (RPGR) localizes in the photoreceptor CC and is believed to function in protein transport in the cilium and disk morphogenesis (34). It was shown to interact with IFT88 and different microtubule proteins (35), and mutations in RPGR account for approximately $20 \%$ of RP cases (22). RPGR, however, is not specific to photoreceptors and some mutations in RPGR also lead to systemic manifestations. Indeed, mutations in RPGR have been associated with primary cilia dyskinesia (PCD), a disorder characterized by primary cilia dysfunction in cells of the respiratory and reproductive systems (36).

Mutations in Retinitis Pigmentosa GTPase Regulator Interacting Protein (RPGRIP), another structural component of the $\mathrm{CC}$ axoneme, have been associated with Leber Congenital Amoursis (LCA), a genetically heterogeneous recessive disorder characterized by severe visual impairment in infants and children. The RPGRIP protein interacts with RPGR at the CC to restrict its localization to the axoneme (37). Dysfunction of RPGRIP causes disorganization of the photoreceptor OS and accumulation of opsin in the IS and cell body, leading to photoreceptor degeneration (34). RPGRIP mutations also affect primary cilium formation in many other cell types in different organs. Consequently, recent studies have shown that mutations in RPGRIP cause Joubert Syndrome type B, a neurodevelopmental autosomal recessive disorder characterized by RP, cerebellar ataxia, and nephronophthisis (NPHP; a renal disease that result in cysts formation in kidneys) (38). When NPHP manifests only with RP, it results in Senior-Loken syndrome. The function of NPHP proteins (NPHP1-6) is not clear, but they have been localized to the CC in photoreceptors, and NPHP4 and RPGRIP have been shown to physically interact, forming a complex that is thought to regulate ciliogenesis (39).

Usher syndrome, in which RP is associated with hearing impairment, is the most frequent syndromic form of RP and the most common cause of deaf-blindness. It has been attributed to mutations in nine different usher genes so far. Interestingly, not all mutations of usher genes lead to deafness and blindness, some mutations result in RP without deafness and vice versa $(40,41)$. The Usher network of proteins belongs to different classes of proteins and performs a wide range 
of functions. It is speculated that the unifying feature is their function in primary cilia. Many of the Usher proteins function as scaffolding proteins and can interact with each other to regulate vesicular transport of proteins to the cilium. In photoreceptors, Usher proteins have been localized to the $\mathrm{CC}$, consistent with a potential role in ciliogenesis. One of the usher genes, $u s h 1 B$, encodes myosin VIIa, which is localized to the site of disk morphogenesis in photoreceptors. In the shaker-1 mouse, a naturally-occurring mutant of $u \operatorname{sh} 1 B$, accumulation of opsin is observed in the IS and CC, suggesting a function for myosin VIIa in protein transport to the $\operatorname{OS}(42,43)$

Another major syndromic form of RP is Bardet-Biedl Syndrome (BBS). BBS is a multisystem disorder, which includes obesity, cognitive impairment, genito-urinary tract malformations, renal anomalies, polydactyl, and retinal degeneration in $90 \%$ of the cases. Protein trafficking defects within the cell body and cilia are thought to cause BBS (44). So far mutations in twelve different genes (BBS1-12) have been associated with BBS (45). The BBS proteins have been localized to the base of the $\mathrm{CC}$ in photoreceptors, close to the basal body where they form a multimeric complex together with Rabin8, a GTP exchange factor for Rab8, which somehow regulates the docking and fusion of vesicles to promote ciliogenesis $(46,47)$. Interestingly, inactivation of Rab8 in photoreceptor cells also leads to accumulation of opsin in the IS and photoreceptor degeneration (21). As the BBS complex appears to interact with a GTP exchange factor for Rab8, these results provide a potential mechanism for the retinal degeneration observed in most BBS cases

\section{CONCLUSIONS}

Although the photoreceptor OS is highly specialized to perform a specific function, it shares common molecular mechanisms that regulate its development and function with other primary cilia. This is reflected, for example, in mutations that cause syndromic forms of RP (Table 1). In fact, $20-30 \%$ of RP patients also display cases of non-ocular disease manifestations, which fall under 30 different syndromes (48). The wide range of disease manifestations in ciliopathies is not surprising considering the diverse roles played by primary cilia. In photoreceptors, 
dysfunction of proteins involved in ciliogenesis and/or protein transport all lead to accumulation of opsin, and possibly other OS-specific proteins, in the IS and cell body. Importantly, this abnormal protein localization appears to cause photoreceptor cell death in most, if not all, cases studied so far (Fig 3). These observations highlight the importance of a better understanding of the mechanisms regulating ciliogenesis and protein trafficking in photoreceptors. Proteomic analysis of the OS and the CC recently revealed a plethora of proteins localized to these cellular compartments (49). These studies provide new candidate proteins that might have a role in ciliogenesis and protein transport in photoreceptors. Future work into the function of these proteins in the development of the OS should shed light on the mechanisms of retinal degenerative diseases, and various other ciliary dysfunctions.

\section{REFERENCES}

1. Barnes AP, Solecki D, Polleux F. New insights into the molecular mechanisms specifying neuronal polarity in vivo. Curr Opin Neurobiol 2008: 18: 44-52.

2. Davenport JR, Yoder BK. An incredible decade for the primary cilium: a look at a onceforgotten organelle. American Journal of Physiology- Renal Physiology 2005: 289: F1159 F1169.

3. Davis EE, Brueckner M, Katsanis N. The Emerging Complexity of the Vertebrate Cilium: New Functional Roles for an Ancient Organelle. Developmental Cell 2006: 11: 9-19. 4. Fliegauf M, Benzing T, Omran H. When cilia go bad: cilia defects and ciliopathies. Nat Rev Mol Cell Biol 2007: 8: 880-893.

5. Insinna C, Besharse JC. Intraflagellar transport and the sensory outer segment of vertebrate photoreceptors. Dev Dyn 2008: 237: 1982-1992.

6. Apfeld J, Kenyon C. Regulation of lifespan by sensory perception in Caenorhabditis elegans. Nature 1999: 402: 804-809.

7. Young RW. The renewal of photoreceptor cell outer segments. The Journal of Cell Biology 1967: 33: 61-72.

8. Kozminski KG, Johnson KA, Forscher P et al. A motility in the eukaryotic flagellum unrelated to flagellar beating. Proceedings of the National Academy of Sciences of the United States of America 1993: 90: 5519-5523.

9. Pedersen LB, Geimer S, Rosenbaum JL. Dissecting the molecular mechanisms of intraflagellar transport in chlamydomonas. Current Biology: CB 2006: 16: 450-459.

10. Sukumaran S, Perkins BD. Early defects in photoreceptor outer segment morphogenesis in zebrafish ift57, ift88 and ift172 Intraflagellar Transport mutants. Vision Research 2009: 49 479-489. 
11. Pazour GJ, Baker SA, Deane JA et al. The intraflagellar transport protein, IFT88, is essential for vertebrate photoreceptor assembly and maintenance. J Cell Biol 2002: 157: 103114.

12. Krock BL, Perkins BD. The intraflagellar transport protein IFT57 is required for cilia maintenance and regulates IFT-particle-kinesin-II dissociation in vertebrate photoreceptors. Journal of cell science 2008: 121: 1907-1915.

13. Ou GS, Blacque OE, Snow JJ et al. Functional coordination of intraflagellar transport motors. Nature (London) 2005: 436 583-587.

14. G. Cole D. Intraflagellar Transport: Keeping the Motors Coordinated. Current Biology 2005: 15: 798

15. Jimeno D, Feiner L, Lillo C et al. Analysis of Kinesin-2 Function in Photoreceptor Cells Using Synchronous Cre-loxP Knockout of Kif3a with RHO-Cre. Invest Ophthalmol Vis Sci 2006: 47: 5039-5046.

16. Marszalek JR, Liu X, Roberts EA et al. Genetic Evidence for Selective Transport of Opsin and Arrestin by Kinesin-II in Mammalian Photoreceptors. Cell 2000: 102: 175-187.

17. Baker SA, Freeman K, Luby-Phelps K et al. IFT20 links kinesin II with a mammalian intraflagellar transport complex that is conserved in motile flagella and sensory cilia. The Journal of Biological Chemistry 2003: 278: 34211-34218.

18. Cole DG, Diener DR, Himelblau AL et al. Chlamydomonas kinesin-II-dependent intraflagellar transport (IFT): IFT particles contain proteins required for ciliary assembly in Caenorhabditis elegans sensory neurons. The Journal of Cell Biology 1998: 141: 993-1008. 19. Blacque OE, Cevik S, Kaplan OI. Intraflagellar transport: from molecular characterisation to mechanism. Frontiers in Bioscience: A Journal and Virtual Library 2008: 13: 2633-2652.

20. Insinna C, Pathak N, Perkins BD et al. The homodimeric kinesin, Kif17, is essential for vertebrate photoreceptor sensory outer segment. Developmental Biology 2008: 316: 160-170.

21. Moritz OL, Tam BM, Hurd LL et al. Mutant rab8 Impairs docking and fusion of rhodopsin-bearing post-Golgi membranes and causes cell death of transgenic Xenopus rods. Molecular Biology of the Cell 2001: 12: 2341-2351.

22. Adams NA, Awadein A, Toma HS. The retinal ciliopathies. Ophthalmic Genet 2007: 28: $113-125$.

23. Humphries MM, Rancourt D, Farrar GJ et al. Retinopathy induced in mice by targeted disruption of the rhodopsin gene. Nature Genetics 1997: 15:216-219.

24. Gao J, Cheon K, Nusinowitz S et al. Progressive photoreceptor degeneration, outer segment dysplasia, and rhodopsin mislocalization in mice with targeted disruption of the retinitis pigmentosa-1 (Rp1) gene. Proceedings of the National Academy of Sciences of the United States of America 2002: 99: 5698-5703.

25. Liu Q, Zuo J, Pierce E. The Retinitis Pigmentosa 1 Protein Is a Photoreceptor Microtubule-Associated Protein. Journal of Neuroscience 2004: 24: 6427-6436.

26. Connell G, Bascom R, Molday L et al. Photoreceptor peripherin is the normal product of the gene responsible for retinal degeneration in the rds mouse. Proc Natl Acad Sci U S A 1991: 88: 723-726.

27. Travis GH, Sutcliffe JG, Bok D. The retinal degeneration slow (rds) gene product is a photoreceptor disc membrane-associated glycoprotein. Neuron 1991: 6: 61-70.

28. Boon CJ, den Hollander AI, Hoyng CB et al. The spectrum of retinal dystrophies caused by mutations in the peripherin/RDS gene. Progress in retinal and eye research 2008: 27: 213-235. 
29. Goldberg AFX. Role of peripherin/rds in vertebrate photoreceptor architecture and inherited retinal degenerations. International Review of Cytology 2006: 253: 131-175.

30. Arikawa K, Molday LL, Molday RS et al. Localization of peripherin/rds in the disk membranes of cone and rod photoreceptors: relationship to disk membrane morphogenesis and retinal degeneration. J Cell Biol 1992: 116: 659-667.

31. Jansen HG, Sanyal S, De Grip WJ et al. Development and degeneration of retina in rds mutant mice: ultraimmunohistochemical localization of opsin. Exp Eye Res 1987: 44: 347-361. 32. Nir I, Papermaster DS. Immunocytochemical localization of opsin in the inner segment and ciliary plasma membrane of photoreceptors in retinas of rds mutant mice. Invest Ophthalmol Vis Sci 1986: 27: 836-840.

33. Sanyal S, De Ruiter A, Hawkins RK. Development and degeneration of retina in rds mutant mice: light microscopy. J Comp Neurol 1980: 194: 193-207.

34. Zhao Y, Hong D-H, Pawlyk B et al. The retinitis pigmentosa GTPase regulator (RPGR)interacting protein: subserving RPGR function and participating in disk morphogenesis.

Proceedings of the National Academy of Sciences of the United States of America 2003: 100: 3965-3970.

35. Wright AF, Shu X. Focus on Molecules: RPGR. Experimental Eye Research 2007: 85: 12.

36. Moore A, Escudier E, Roger G et al. RPGR is mutated in patients with a complex X linked phenotype combining primary ciliary dyskinesia and retinitis pigmentosa. Journal of Medical Genetics 2006: 43: 326-333.

37. Hong DH, Yue G, Adamian M et al. Retinitis pigmentosa GTPase regulator (RPGRr)interacting protein is stably associated with the photoreceptor ciliary axoneme and anchors RPGR to the connecting cilium. The Journal of Biological Chemistry 2001: 276: 12091-12099. 38. Arts HH, Doherty D, van Beersum SEC et al. Mutations in the gene encoding the basal body protein RPGRIP1L, a nephrocystin-4 interactor, cause Joubert syndrome. Nature Genetics 2007: 39: 882-888.

39. Roepman R, Letteboer SJ, Arts HH et al. Interaction of nephrocystin-4 and RPGRIP1 is disrupted by nephronophthisis or Leber congenital amaurosis-associated mutations. Proceedings of the National Academy of Sciences of the United States of America 2005: 102: 18520-18525.

40. Williams DS. Usher syndrome: Animal models, retinal function of Usher proteins, and prospects for gene therapy. Vision Research 2008: 48 433-441.

41. Reiners J, Nagel-Wolfrum K, Jürgens K et al. Molecular basis of human Usher syndrome: deciphering the meshes of the Usher protein network provides insights into the pathomechanisms of the Usher disease. Experimental Eye Research 2006: 83: 97-119.

42. Weil D, Blanchard S, Kaplan J et al. Defective myosin VIIA gene responsible for Usher syndrome type 1B. Nature 1995: 374: 60-61.

43. Liu X, Udovichenko IP, Brown SDM et al. Myosin VIIa Participates in Opsin Transport through The Photoreceptor Cilium. Journal of Neuroscience 1999: 19: 6267-6274.

44. Blacque OE, Leroux MR. Bardet-Biedl syndrome: an emerging pathomechanism of intracellular transport. Cellular and molecular life sciences : CMLS 2006: 63: 2145-2161.

45. Tobin JL, Beales PL. Bardet-Biedl syndrome: beyond the cilium. Pediatr Nephrol 2007: 22: $926-936$.

46. Leroux M. Taking Vesicular Transport to the Cilium. Cell 2007: 129: 1041-1043.

47. Nachury M, Loktev A, Zhang Q et al. A Core Complex of BBS Proteins Cooperates with the GTPase Rab8 to Promote Ciliary Membrane Biogenesis. Cell 2007: 129: 1201-1213. 
48. Hartong D, Berson E, Dryja T. Retinitis pigmentosa. The Lancet 2006: 368: 1795-1809. 49. Liu Q, Tan G, Levenkova $\mathrm{N}$ et al. The proteome of the mouse photoreceptor sensory cilium complex. Mol Cell Proteomics 2007: 6: 1299-1317. 


\section{FIGURE LEGENDS}

Figure 1. The vertebrate photoreceptor. (A) An illustration of the photoreceptor structure. The photosensitive region (OS) is composed of stacks of disk membranes that are linked to the inner segment (IS) by the connecting cilium (CC). The nucleus (N) and synaptic terminals (ST) are also illustrated. (B) An illustration of the OS disk membrane domains and the specific proteins they contain. (C) An illustration focusing on the connecting cilium area, a region of disk morphogenesis and protein transport. The doublet microtubule arrangement at the CC level gives way to singlet microtubules arrangement in the distal OS. RPE: Retinal Pigmented Epithelium.

Figure 2. Intraflagellar transport in photoreceptors. A simplified illustration of intraflagellar transport (IFT) at the connecting cilium level of the vertebrate photoreceptor. The IFT motors, heterotrimeric kinesin II and dynein are used for anterograde and retrograde transport along the axoneme, respectively. The motor proteins transport cargo such as rhodopsin in association with the IFT complex.

Figure 3. Protein trafficking or ciliogenesis defects in photoreceptors lead to cell death. (A) In a wildtype photoreceptor, cargo proteins such as rhodopsin are trafficked to the outer segment (OS) through the connecting cilium (CC). The green arrow represents normal trafficking in the CC. (B) Mutations that affect photoreceptor protein trafficking, or ciliogenesis, lead to accumulation of rhodopsin in the inner segment (IS) and cell body, which eventually result in photoreceptor cell death. N - Nucleus, ST - Synaptic Terminal. 
A

B

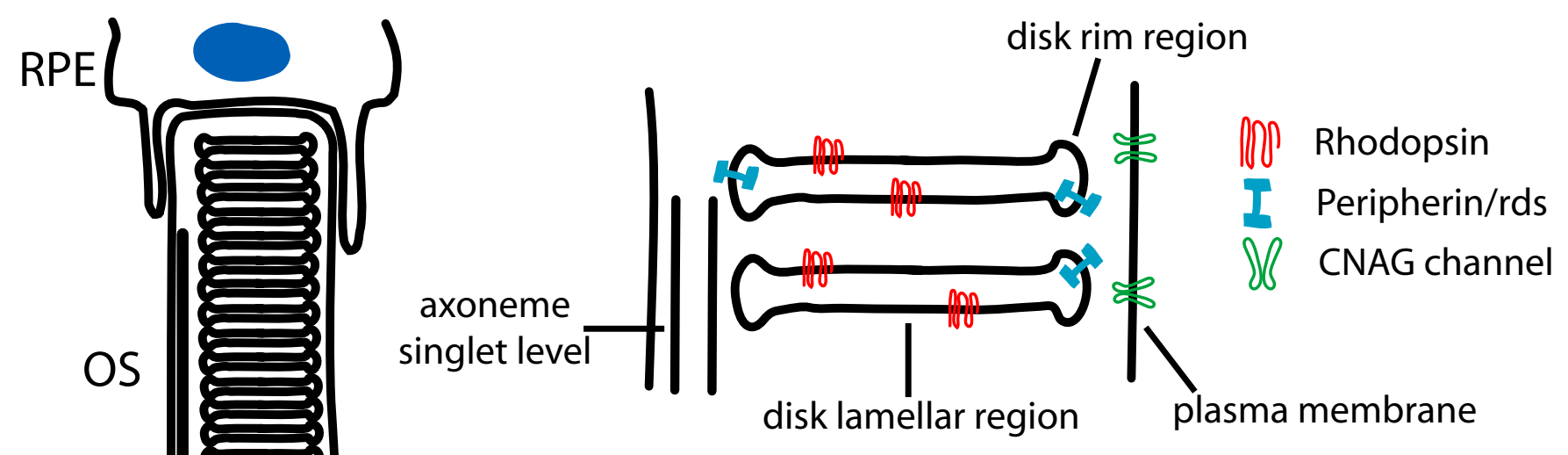

CC
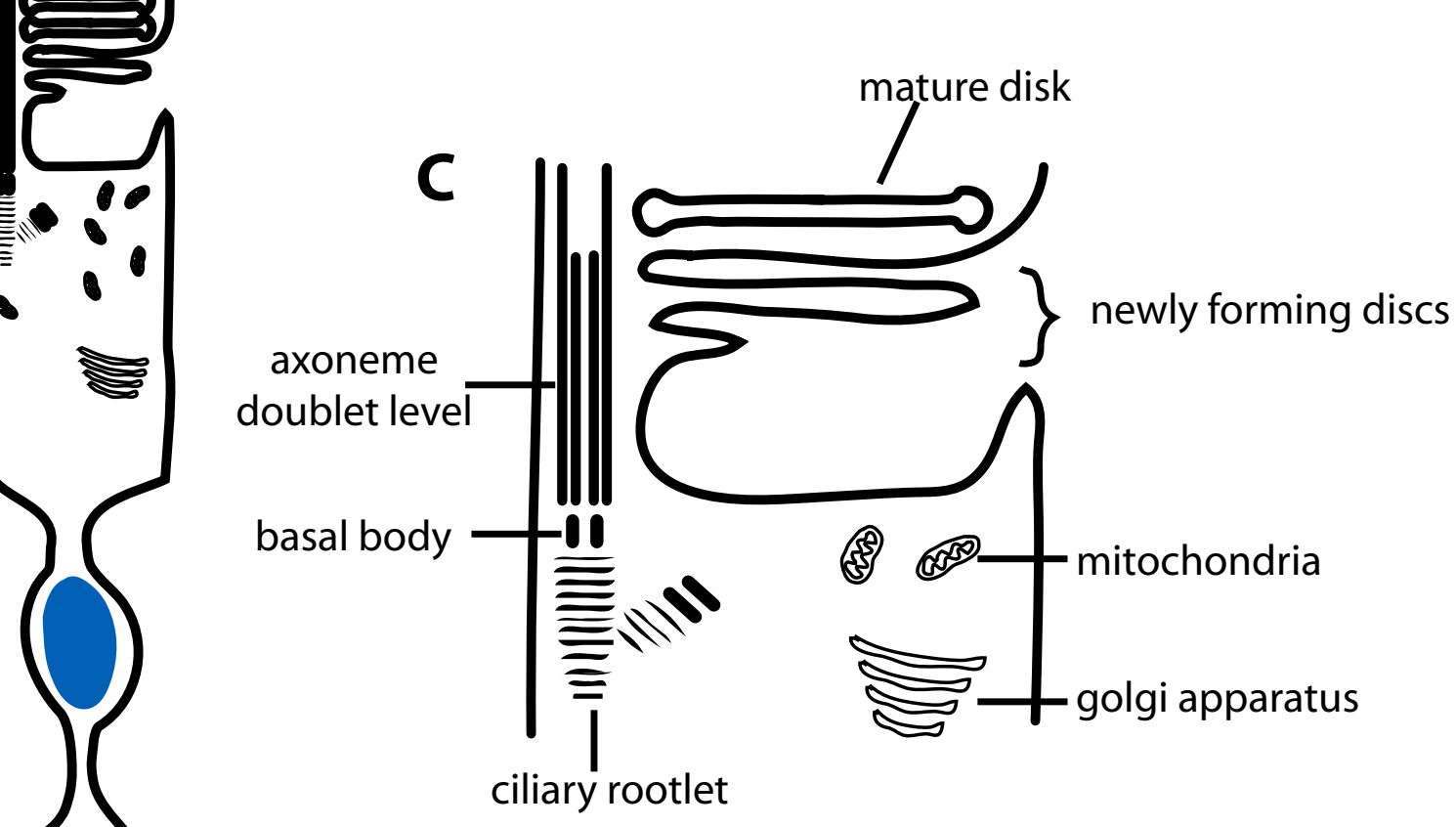

ST

IS

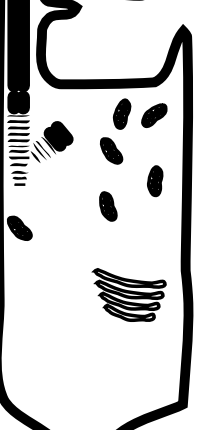

$\mathrm{N}$
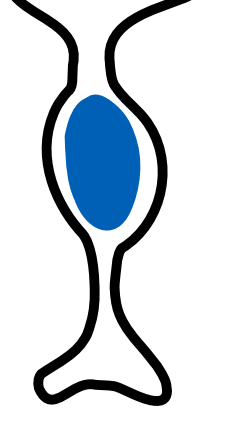

ciliary rootlet

Figure 1. 

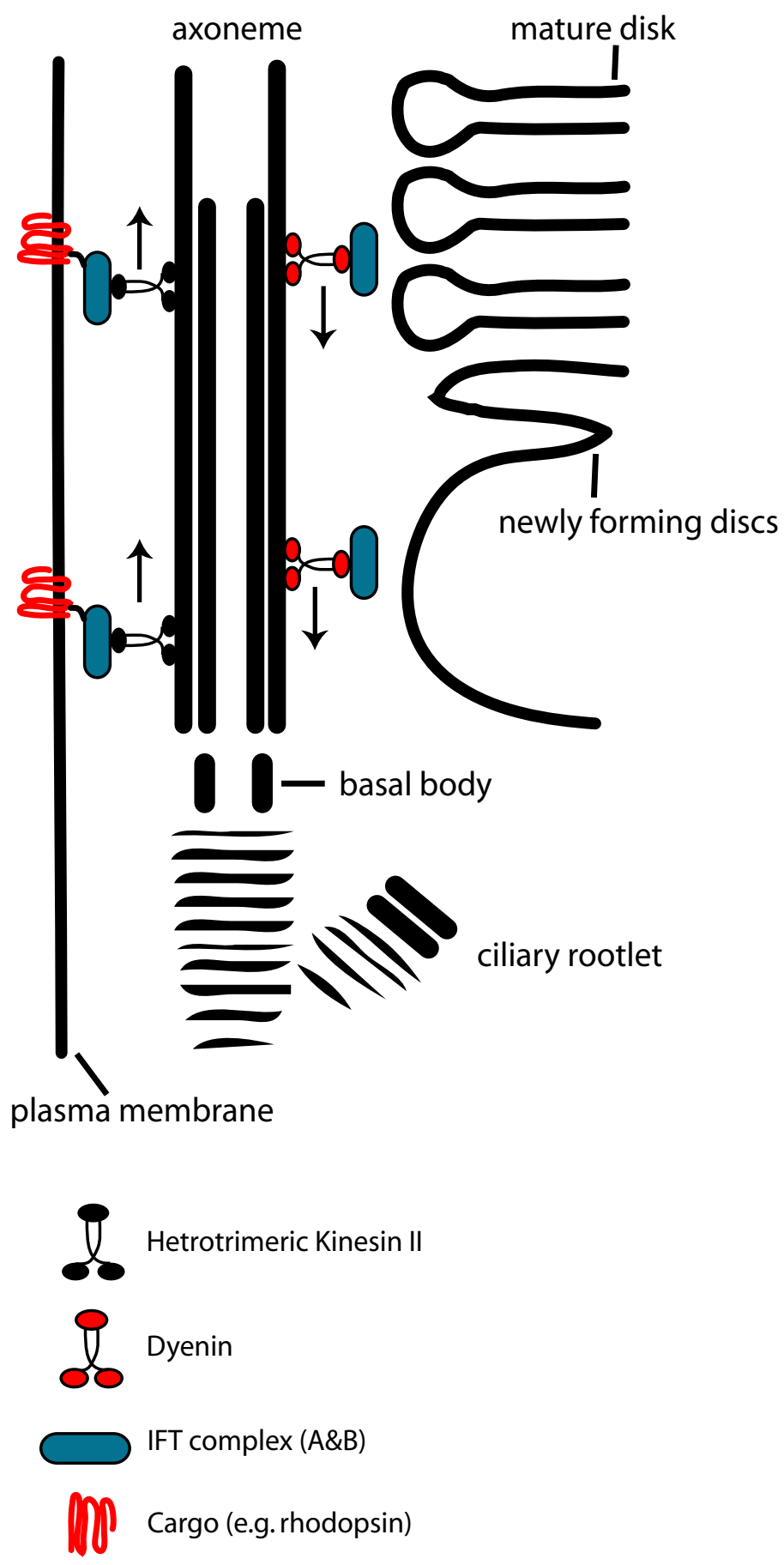

Figure 2. 
Figure 3.

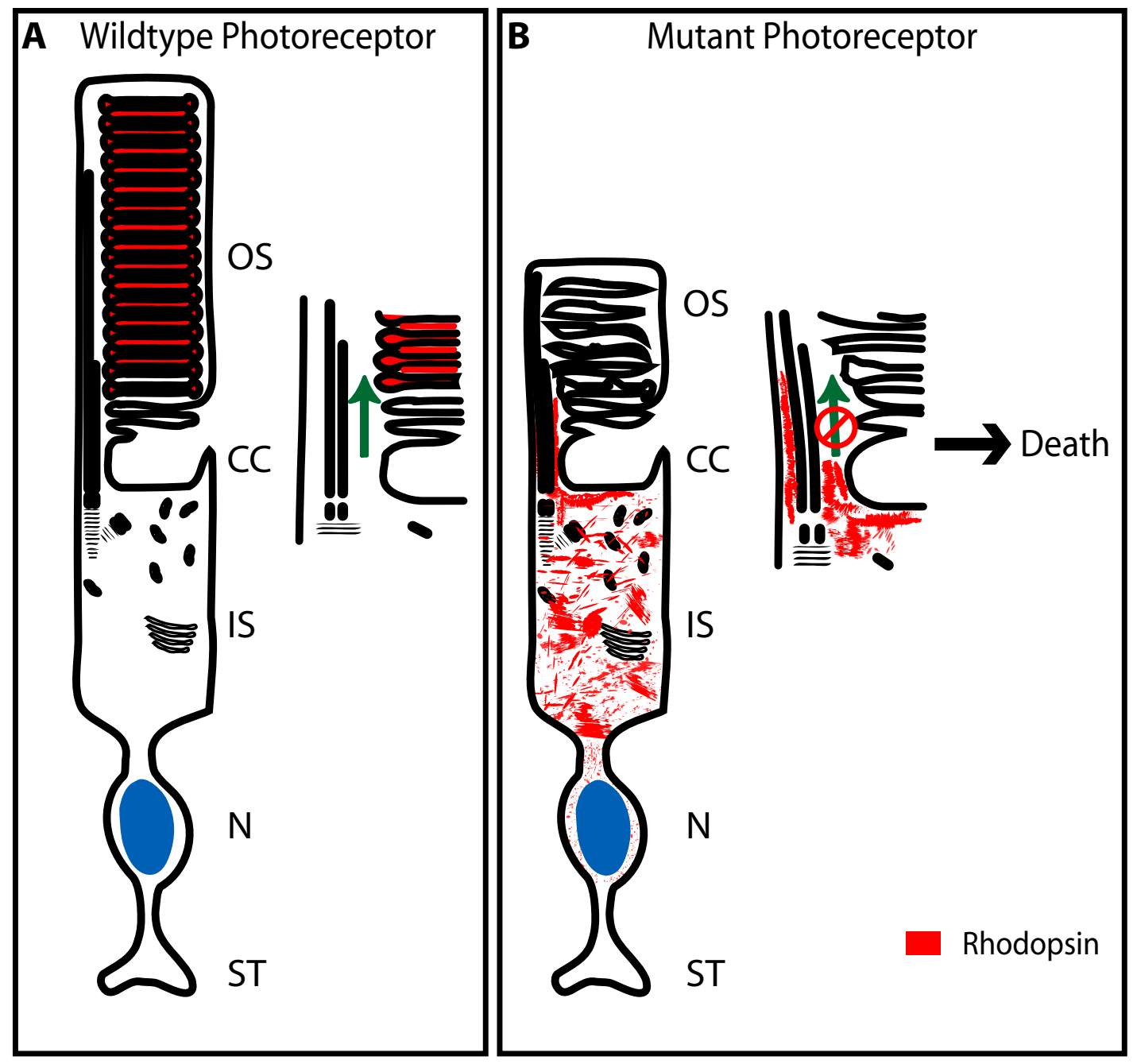

